other supports in their communities (e.g. family, school, and other parents of children living with type 1 diabetes). Parents described the process of learning how to manage type 1 diabetes with many relying on the diabetes team as a source of information. Most parents noted that how they felt about their child's type 1 diabetes had changed over time; however many stated that being involved in their child's self-management of type 1 diabetes continued to have an impact of their own well-being.

Conclusions It is important that parents are supported as they deal with their own emotions in relation to their child's type 1 diabetes diagnosis. Understanding the impact of a type 1 diabetes diagnosis on parents may enable healthcare professionals to provide more effective support to parents as they come to terms with their child's diagnosis and learn to manage type 1 diabetes. Ensuring that parents are supported as their role in their child's type 1 diabetes management changes over time may subsequently impact the negotiation of self-management between parents and adolescents as adolescents take on increasing levels of responsibility.

\section{P237 AN ASSESSMENT OF CONFIDENCE AND KNOWLEDGE REGARDING SELF-MANAGEMENT OF TYPE I DIABETES AMONGST ADOLESCENTS ATTENDING A DIABETES TRANSITION CLINIC}

${ }^{1}$ Kerrie Hennigan*, ${ }^{1} \mathrm{~A}$ Quinn, ${ }^{1} \mathrm{M}$ Norris, ${ }^{1} \mathrm{M}$ Conroy-Kelly, ${ }^{1,2}$ Maaz Mirza, ${ }^{1}$ Orla M Neylon, ${ }^{1}$ Eoin Noctor, ${ }^{1}$ Clodagh O'Gorman. 'UHL, Limerick, Ireland; ${ }^{2}$ Graduate Entry Medical School, Limerick, Ireland

10.1136/archdischild-2019-epa.587

Aims To collate the questionnaire responses of adolescents with Type I Diabetes Mellitus (T1DM) who attended a transition clinic between paediatric and adult diabetes care in University Hospital Limerick (UHL) and to analyse their confidence levels in specific parts of their diabetes management (hypoglycaemia, insulin dose adjustment, carbohydrate counting) and their knowledge levels around sexual health, alcohol safety, safe driving and smoking.

Results $100 \%$ of patients said their expectations of the clinic were met. $100 \%$ of patients felt that they had adequate information and education on the management of hypoglycaemia, high glucose and ketones. $14 \%$ felt they did not have adequate information on insulin dose adjustment and carbohydrate counting. When it came to adolescent specific topics, $100 \%$ of patients felt they had adequate information on the effects of alcohol and smoking on diabetes management, with $29 \%$ stating they did not have adequate knowledge on sexual health and safe driving education prior to this clinic. $86 \%$ of patients said that they felt 'more confident' about transitioning to adult diabetes care after attending this transition clinic.

Conclusion The transition from childhood to adulthood can be a difficult and turbulent period for any child, but especially more so for those with T1DM. Along with increased autonomy comes an increased risk of poor glycaemic control due to changes in health needs, management, and providers. This study allows us to assess the need for and the benefits gained from this new transition clinic initiative whilst also highlighting areas where diabetes education may be lacking in some adolescents, allowing us to fill these knowledge gaps prior to their transition to adult diabetes care.

\section{P238} EXERCISE AND ACTIVITIES UNDERTAKEN BY CHILDREN WITH TYPE 1 DIABETES MELLITUS (T1DM)

Niall Dalton*, Julie Evers, Madalene Khalil, Orla Neylon, Paul Scully, Clodagh O'Gorman. University Hospital Limerick, Limerick, Ireland

\subsection{6/archdischild-2019-epa.588}

Aims A physically active lifestyle helps with managing diabetes by improving cardiovascular fitness, increasing insulin sensitivity, improving school performance, cognition, and enhancing quality of life. However, despite efforts to promote an active lifestyle, a significant proportion of children with T1DM remain inactive. The aim of this study was to examine exercise and activities undertaken by young people with T1DM.

Methods The study was a regional level observational clinical trial within the University Hospital Limerick T1DM outpatient clinic, focusing on self-reporting of activity using the Children's sport participation and physical activity study (CSPPA) questionnaire. Questionnaires were completed between June and August 2018 and analysed using SPSS statistical software.

Results 55 children completed the survey. 55\% of respondents were female and age range was from 5 - 17 years, with all respondents being diagnosed a minimum of 2 years previously. $64 \%$ of respondents were based in a rural location. Almost two-thirds of respondents undertook between 60 and $180 \mathrm{~min}$ of physical activity per weekday. $83 \%$ of respondents watched television with one-third watching daily. $57 \%$ of respondents used computers with two-thirds daily. $20 \%$ of respondents used a mobile phone with one-third using daily. $83 \%$ of respondents travelled to school by car or bus with two-thirds living within $5 \mathrm{k}$ of school. The most common reason for using a car or bus was for road safety reasons with only $4 \%$ stating becoming hypoglycaemic was an issue.

Conclusion The results from this study show that there was a variable level of activity amongst children with T1DM. The majority watched television on a daily basis while most were transported to school on the basis of road safety. Future work in this area should be to further promote undertaking activity, including the provision of schemes to help promote activities such as walking or cycling to school.

\section{P239 QUALITY OF OUTPATIENT CARE RECEIVED BY CHILDREN AND YOUNG PEOPLE WITH TYPE 1 DIABETES MELLITUS (T1DM) IN A PERIPHERAL HOSPITAL}

${ }^{1}$ Deirdre O'Sullivan*, Ryan O'Dowling ${ }^{2},{ }^{1}$ Charmaine Van Heerden, ${ }^{3}$ Akhtar Khan ${ }^{1}$ University Hospital Kerry, Tralee, Ireland; ' ${ }^{2}$ University Hospital Kerry, Tralee, Ireland; ${ }^{3}$ University Hospital Kerry, Tralee, Ireland

\subsection{6/archdischild-2019-epa.589}

Introduction Type 1 Diabetes Mellitus (T1DM) is a common health condition affecting children and young people. T1DM is managed largely in the outpatient setting. Regular and ongoing outpatient care is essential to maintain optimal glycemic control and to monitor for complications.

Aims The objective of this study was (1) to examine the quality of outpatient care received by children and young people with T1DM in a peripheral hospital and (2) to suggest ways to improve the service in peripheral hospitals in Ireland. A reaudit could be done after implementation of such suggestions. Methods This was a retrospective study where patients (0 to 16 years old) with T1DM were identified by an electronic search of the Hospital In-Patient Enquiry (HIPE) system 